\title{
Magnetic excitations of carbon nanotubes in chiral model of graphene
}

\author{
Yu.P. Rybakov ${ }^{1, \star}$ \\ ${ }^{1}$ RUDN University, Moscow, Russian Federation
}

\begin{abstract}
Within the scope of the chiral model of graphene in 8-spinor realization, suggested earlier [1, 2], the combined spinor field $\Psi$ is used as a new order parameter, which contains information on spin and quasispin excitations in graphene (8-spinor part) and also that on $s p$-hybridization effect of carbon valence electrons [SU(2)-matrix part]. This model admits two kinds of configurations describing, in the long-wave approximation, the graphene mono-atomic plane and the carbon nanotube respectively. The first one is given by the domain-wall (or kink-like) solution and the second one is described by the hedgehog ansatz with large topological charge (winding number $n$ ). We consider the infinite carbon nanotube in a static external magnetic field oriented along the tube and analyze the structure of the magnetic intensity on the outside. The diamagnetic behavior of the material is shown by the perturbation method which proves to be self-consistent for $n \gg 1$.
\end{abstract}

\section{Introduction. Structure of the model}

The discovery of carbon nanotubes [3, 4], which are endowed with very high electric and heat conductivities and also with an extraordinary tensile and bending strength, opened new possibilities in spintronics and in production of composite materials for their reinforcing. This fact stimulated a large number of theoretical studies on the electronic properties of carbon nanotubes. The magnetic properties of carbon nanotubes were studied by H. Ajiki and T. Ando [5, 6] within the scope of the effective-mass theory. These authors found that in a magnetic flux parallel to the tube axis the magnetic moment oscillated as a function of the flux. For a magnetic field perpendicular to the axis carbon nanonubes exhibited a strong diamagnetism. Large diamagnetic susceptibility was found experimentally for a magnetic field both perpendicular and parallel to the tube axis [7,8].

We suggest here a phenomenological model which permits to calculate all necessary characteristics of both carbon nanotubes and other possible graphene-like configurations. The concept behind this research is the following. The carbon atom possesses of four valence electrons in the so-called hybridized $s p^{2}$-states. The one of them is "free" in the honeycomb graphene lattice and all others form the strong covalent bonds with the neighbors. It appears natural to introduce scalar $a_{0}$ and 3-vector $\vec{a}$ fields corresponding to the $s$-orbital and the $p$-orbital states of the "free" electron respectively. These two fields can be combined into the unitary matrix

$$
V=a_{0} \tau_{0}+l \vec{a} \cdot \vec{\tau}
$$

considered as the order parameter of the model, the longwave approximation being adopted, where $\tau_{0}$ is the unit

\footnotetext{
^e-mail: rybakov_yup@pfur.ru
}

$2 \times 2$-matrix and $\vec{\tau}$ are the three Pauli matrices, with the $S U(2)$-condition

$$
a_{0}^{2}+\vec{a}^{2}=1
$$

being imposed. The model admits the kink-like configuration

$$
V=\exp \left[\imath \tau_{3} \Theta(z)\right]
$$

of the graphene plane and the hedgehog one of the carbon nano-tube [1]:

$$
\begin{array}{r}
V=\exp [l \sigma \Theta(r)], \quad \sigma=\tau_{1} \cos \phi+\tau_{2} \sin \phi, \\
\phi=n \varphi, n=1,2 \ldots,
\end{array}
$$

with $r, \varphi, z$ being polar coordinates and $\Theta$ designating the chiral angle. Here $n$ stands for the topological charge $Q$ of the degree type which is known as the helicity or the winding number:

$$
Q=\operatorname{deg}\left\{S^{2} \rightarrow S^{2}\right\}=\frac{1}{4 \pi} \int_{\mathbb{R}^{2} \cup \infty} d \phi d \Theta \sin \Theta=n .
$$

In the sequel the helicity $n$ will be chosen positive.

For the description of spin and quasi-spin excitations, the latter ones corresponding to the independent excitation modes of the two triangular sub-lattices, we introduce the two Dirac spinors $\psi_{1}, \psi_{2}$ and consider the combined spinor field $\Psi$ as a new order parameter:

$$
\Psi=\xi \otimes\left(\psi_{1} \oplus \psi_{2}\right),
$$

where $\xi$ stands for the first column of the matrix $V$. The Lagrangian density $L$ of the model

$$
\begin{aligned}
L=\frac{I}{2} \overline{D_{\mu} \Psi} P D^{\mu} \Psi & -\frac{\lambda^{2}}{2} \vec{a}^{2} j_{\mu} j^{\mu}+ \\
& +\iota \mu_{0} \vec{a}^{2} \bar{\Psi} \sigma_{\mu \nu} F^{\mu \nu} \Psi-\frac{1}{16 \pi} F_{\mu \nu} F^{\mu \nu}
\end{aligned}
$$


contains the projector $P=\gamma^{v} j_{v}$ on the positive energy states, where $j_{\mu}=\bar{\Psi} \gamma_{\mu} \Psi, \mu=0,1,2,3$, designates the Dirac current, $\bar{\Psi}=\Psi^{+} \gamma_{0}, \gamma_{\mu}$ stands for the Dirac matrices. Here standard denotations are introduced for the Pauli antisymmetric spin operator

$$
\sigma_{\mu \nu}=\left[\gamma_{\mu}, \gamma_{v}\right] / 4
$$

and for the electromagnetic field tensor $F_{\mu \nu}=\partial_{\mu} A_{\nu}-\partial_{\nu} A_{\mu}$, the constant $\mu_{0}>0$ denoting the Bohr magneton per lattice spacing cubed. The model contains the two constant parameters: the exchange energy $I$ per lattice spacing $a$ and some characteristic inverse length $\sqrt{\lambda}$. The interaction with the electromagnetic field is realized through the extension of the derivative:

$$
D_{\mu}=\partial_{\mu}-\imath e_{0} A_{\mu} \Gamma_{e},
$$

with $e_{0}>0$ being the coupling constant and $\Gamma_{e}=\left(1-\tau_{3}\right) / 2$ being the charge operator chosen in accordance with the natural boundary condition at infinity: $a_{0}(\infty)=1$. However, the additional interaction term of the Pauli type should be added to take into account the proper magnetic moments of the electrons.

The comparison of the Lagrangian (3) with that of the Landau-Lifshitz theory shows the proportionality of the parameter $I$ to the exchange energy $E_{\text {exch }}$ between atoms and also the following structure of the coupling constants $e_{0}$ and $\mu_{0}$ :

$$
I=\frac{E_{\text {exch }}}{a}, \quad e_{0}=\frac{e}{\hbar c}, \quad \mu_{0}=\frac{e \hbar}{2 m_{e} c a^{3}},
$$

where the exchange energy is usually adopted as $E_{\text {exch }}=$ $2,9 \mathrm{eV}$ and the lattice spacing as $a=3,56 \cdot 10^{-8} \mathrm{~cm}$, with $e$ being the absolute value of the electron charge. Finally, one can find the following numerical values of the parameters in question:

$$
e_{0} I=2 \cdot 10^{3} \text { Gauss, } \quad \mu_{0}=2 \cdot 10^{2} \text { Gauss. }
$$

Within the frame of the graphene chiral model we investigated earlier the magnetic excitations of the graphene plane. In particular, magnetic properties of the graphene plane appear to be highly anisotropic: for the case of an external magnetic field parallel to the plane the material reveals diamagnetic behavior, however, for the case of the orthogonal field orientation it reveals paramagnetic behavior [9]. It is worth-while to stress that similar behavior is predicted for the bilayer graphene $[10,11]$. These results are in agreement with experiments [12].

We consider now an infinite carbon nanotube interacting with an external static magnetic field oriented along the tube. The axially-symmetric configuration being studied, we analyze the structure of the corresponding solution via the perturbation method. This method proves to be self-consistent under the supposition that the topological charge of the tube is large. Using the Green function method, we find the magnetic intensity of the tube and show the field to decrease in the central domain.

\section{Axially-symmetric configuration}

Let us describe first the static magnetic field through the azimuth component of the vector potential:

$$
A_{\varphi}=A(r), \quad B_{3}=B=\frac{1}{r} \partial_{r}(r A),
$$

with the natural boundary condition at infinity:

$$
A(r \rightarrow \infty)=\frac{1}{2} B_{0} r, \quad B_{0}=\text { const }
$$

It is also convenient to use the tangential basis of the Dirac $\gamma$-matrices in 8-spinor realization:

$$
\begin{array}{r}
\gamma_{0}=\tau_{0} \otimes \tau_{1} \otimes \tau_{0} ; \gamma_{1}=-l \lambda_{1} \otimes \tau_{2} \otimes \tau_{0} ; \\
\gamma_{2}=-\imath \lambda_{2} \otimes \tau_{2} \otimes \tau_{0} ; \gamma_{3}=-l \lambda_{3} \otimes \tau_{2} \otimes \tau_{0},
\end{array}
$$

where

$\lambda_{1}=\tau_{1} \cos \varphi+\tau_{2} \sin \varphi ; \lambda_{2}=\tau_{2} \cos \varphi-\tau_{1} \sin \varphi ; \lambda_{3}=\tau_{3}$

Due to this choice of the basis, the spinor connection vanishes in the plane case and one finds

$$
\sigma_{12}=\frac{1}{4}\left[\gamma_{1}, \gamma_{2}\right]=-l \frac{r}{2} \tau_{3}
$$

As can be seen from (3), the model admits the mirror symmetry

$$
\Psi \Rightarrow \gamma_{0} \Psi
$$

and also the quasi-spin symmetry

$$
\psi_{1} \Leftrightarrow \psi_{2},
$$

with the spinor invariant field being

$$
\psi_{1}=\psi_{2}=\psi=\operatorname{col}(w, w)
$$

where $w$ stands for the 2-spinor. The structure (6) indicates that the spatial Dirac current vanishes: $\vec{j}=\bar{\Psi} \vec{\gamma} \Psi=0$. On the other hand, if one takes into account that the electron spin should be oriented contra magnetic field, the new combined symmetry group can be revealed:

$$
G=\operatorname{diag}\left[S O(2)_{S} \otimes U(1)\right],
$$

where the rotations around $z$-axis are combined with the $U(1)$-transform: $w \Rightarrow \exp (\imath \beta) w$. Therefore, the $G$ invariance yields the following equation for $w$ :

$$
J_{3} w=-\imath \partial_{\varphi} w+\frac{1}{2} \tau_{3} w=-\frac{1}{2} w
$$

where $J_{3}$ stands for the rotation generator. We infer that the solution to the equation (7) takes the form

$$
w=\operatorname{col}\left[w_{1}(r) \exp (-l \varphi), w_{2}(r)\right]
$$

where the correspondence with the scalar chiral model implies the boundary condition at $r \rightarrow \infty: j_{\mu} j^{\mu}(\infty)=1$ or

$$
w_{1} \rightarrow 0, \quad\left|w_{2}\right|^{2} \rightarrow \frac{1}{4}
$$


Inserting (8) into the Lagrangian (3) and taking into account the structure (1) of the carbon nanotube and the boundary condition (9), we confine our attention to the simplest possible configuration $w_{1}=0, w_{2}^{*}=w_{2}$. As a result, we obtain the following effective Lagrangian density:

$$
\begin{gathered}
L=-8 I\left[U^{2}\left(\partial_{r} \Theta\right)^{2}+\frac{1}{4}\left(\partial_{r} U\right)^{2}+\right. \\
\left.+\frac{1}{r^{2}}\left(n+e_{0} r A\right)^{2} U^{2} \sin ^{2} \Theta\right]- \\
-8 \lambda^{2} U^{2} \sin ^{2} \Theta+\frac{2 \mu_{0}}{r} U \sin ^{2} \Theta \partial_{r}(r A)- \\
\quad-\frac{1}{8 \pi r^{2}}\left(\partial_{r}(r A)\right)^{2},
\end{gathered}
$$

where $U=w_{2}^{2}$ and the boundary conditions are imposed:

$$
U(\infty)=\frac{1}{4}, \quad \Theta(\infty)=0, \quad \Theta(-\infty)=\pi
$$

Changing the independent variable $r=\ell e^{\eta}$, where $\ell=$ $\sqrt{I} / \lambda$, let us first consider the asymptotic domain $\eta \rightarrow \infty$ through the substitution:

$$
U=\frac{1}{4}+\zeta, \quad A=\frac{1}{2} B_{0} \ell e^{\eta}+\alpha, \quad \Theta=2 \arctan R,
$$

with $\zeta, \alpha, R$ vanishing at infinity. One derives from (10) and (12) the following equation for $R$ :

$$
\begin{aligned}
& R^{\prime \prime}\left(1+R^{2}\right)-2 R R^{\prime 2}=R\left(1-R^{2}\right) \times \\
& \quad \times\left[\left(n+e_{0} B_{0} \ell^{2} e^{2 \eta} / 2\right)^{2}+e^{2 \eta}\left(1-\mu_{0} B_{0} \ell^{2} / I\right)\right] .
\end{aligned}
$$

By inspection that $n \gg 1$ one can find the approximate solution to the equation (13) of the form:

$$
R \approx k \exp \left(-n \eta-m e^{2 \eta}\right)
$$

where $4 m=e_{0} B_{0} \ell^{2}$, and the constant $k$ determines the radius $r_{0}$ of the nanotube according to the formula $k^{2}=$ $r_{0} / \ell$. It is worth-while to notice that the helicity $n$ has no any relation to the number $N$ of walls. In fact, for $N>1$ the substitution (12) for $\Theta$ should be replaced with $\Theta=$ $2 N \arctan R$.

Let us study now the magnetic field structure through the perturbation method. To this end, we consider (14) as the source for the perturbation $\alpha$ of the vector potential satisfying the equation:

$$
\alpha^{\prime \prime}-\alpha=2 \pi \ell\left(\mu_{0}-e_{0} I\right)\left(\sin ^{2} \Theta\right)^{\prime} \equiv j(\eta) .
$$

The corresponding magnetic intensity $B=B_{0}+b$ can be found from (15) by the Green's function method. In our case the Green's function reads:

$$
G\left(\eta, \eta^{\prime}\right)=-\frac{1}{2} \begin{cases}e^{\eta^{\prime}-\eta}, & \text { if } \eta^{\prime}<\eta \\ e^{\eta-\eta^{\prime}}, & \text { if } \eta^{\prime}>\eta\end{cases}
$$

and the solution to (15) acquires the form:

$$
\alpha(\eta)=-\frac{1}{2} \int_{-\infty}^{\eta} d \eta^{\prime} e^{\eta^{\prime}-\eta} j\left(\eta^{\prime}\right)-\frac{1}{2} \int_{\eta}^{\infty} d \eta^{\prime} e^{\eta-\eta^{\prime}} j\left(\eta^{\prime}\right) .
$$

Taking into account that $b=\ell^{-1} e^{-\eta}\left(\alpha+\alpha^{\prime}\right)$, one obtains from (15) and (16) the perturbation part of the magnetic intensity:

$$
b=-\ell^{-1} \int_{\eta}^{\infty} d \eta^{\prime} e^{-\eta^{\prime}} j\left(\eta^{\prime}\right)=2 \pi\left(\mu_{0}-e_{0} I\right) \sin ^{2} \Theta .
$$

Numerical estimates, based on the correspondence with the Landau-Lifshitz theory, show that $e_{0} I \gg \mu_{0}$. Thus, in virtue of (17), the magnetic intensity decreases inside the nanotube, with the susceptibility $\chi=b / B_{0}$ being proportional to the radius of the nanotube since $\sin ^{2} \Theta \propto k^{2}=$ $r_{0} / \ell$. This result is confirmed experimentally [7].

\section{Conclusion}

We applied the chiral model of graphene in 8-spinor realization to the description of the infinite carbon nanotube interacting with the static magnetic field oriented along the tube. We construct the approximate solution to the model equations, which proves to be valid asymptotically for the case of the tube endowed with the topological charge $n \gg 1$. We find the magnetic intensity outside the tube by applying the perturbation scheme based on this solution and show the diamagnetic behavior of the material. As can be seen from (3) and the structure of the extended derivative $D_{\mu}$, the predicted magnetically insulating properties of carbon nanotubes seem to be emerging from the superconductive behavior of carbon structures.

\section{Acknowledgement}

This work was financially supported by the Ministry of Education and Science of the Russian Federation (the Agreement number 02.a03.21.0008).

\section{References}

[1] Yu.P. Rybakov, Solid State Phenomena 190, 59-62 (2012)

[2] Yu.P. Rybakov, Solid State Phenomena 233-234, 1619 (2015)

[3] S. Iijima, Nature 354, 56-58 (1991)

[4] S. Iijima and T. Ichihashi, Nature 363, 603-605 (1993)

[5] H. Ajiki and T. Ando, J. Phys. Soc. Jap. 62, 2470-2480 (1993)

[6] H. Ajiki and T. Ando, J. Phys. Soc. Jap. 64, 4382-4391 (1995)

[7] X.K. Wang et al., J. Mater. Res. 9, 1578-1590 (1994)

[8] A.P. Ramirez et al., Science 265, 84-86 (1994)

[9] Yu.P. Rybakov, M. Iskandar, A.B. Ahmed, RUDN J. of Math. Inform. Physics 25, Issue 3, 266-275 (2017)

[10] Yu.P. Rybakov, A.B. Ahmed, Intern. J. of Appl. Math. and Theor. Physics 3, Issue 3, 74-77 (2017)

[11] Yu.P. Rybakov, A.B. Ahmed, S. Massou and M. Iskandar, Far East J. Math. Sci. 102, Issue 2, 399-407 (2017)

[12] M. Sepioni et al., Phys. Rev. Lett. 105, 207205 (2010) 